OPEN ACCESS

Edited by:

Dario Leister

Ludwig Maximilian University

of Munich, Germany

Reviewed by:

Bernhard Grimm

Humboldt University of Berlin,

Germany

Poul Erik Jensen,

University of Copenhagen, Denmark

*Correspondence:

Tatsuru Masuda

ctmasuda@fye.c.u-tokyo.ac.jp

tPresent address:

Nino A. Espinas,

Plant Epigenetics Unit, Okinawa Institute of Science and Technology Graduate University, Okinawa, Japan

Specialty section:

This article was submitted to

Plant Physiology,

a section of the journal

Frontiers in Plant Science

Received: 01 June 2016 Accepted: 18 August 2016 Published: 31 August 2016

Citation:

Espinas NA, Kobayashi K, Sato $Y$, Mochizuki N, Takahashi K, Tanaka $R$

and Masuda T (2016) Allocation

of Heme Is Differentially Regulated by

Ferrochelatase Isoforms

in Arabidopsis Cells.

Front. Plant Sci. 7:1326.

doi: 10.3389/fpls.2016.01326

\section{Allocation of Heme Is Differentially Regulated by Ferrochelatase Isoforms in Arabidopsis Cells}

\author{
Nino A. Espinas ${ }^{1 \dagger}$, Koichi Kobayashi ${ }^{2}$, Yasushi Sato ${ }^{3}$, Nobuyoshi Mochizuki \\ Kaori Takahashi ${ }^{5}$, Ryouichi Tanaka ${ }^{5}$ and Tatsuru Masuda ${ }^{1,2 *}$
}

${ }^{1}$ Graduate School of Science, The University of Tokyo, Tokyo, Japan, ${ }^{2}$ Graduate School of Arts and Sciences, The University of Tokyo, Tokyo, Japan, ${ }^{3}$ Graduate School of Science and Engineering, Ehime University, Ehime, Japan, ${ }^{4}$ Graduate School of Science, Kyoto University, Kyoto, Japan, ${ }^{5}$ Institute of Low Temperature Science, Hokkaido University, Sapporo, Japan

Heme is involved in various biological processes as a cofactor of hemoproteins located in various organelles. In plant cells, heme is synthesized by two isoforms of plastid-localized ferrochelatase, FC1 and FC2. In this study, by characterizing Arabidopsis T-DNA insertional mutants, we showed that the allocation of heme is differentially regulated by ferrochelatase isoforms in plant cells. Analyses of weak $\left(f_{c} 1-1\right)$ and null (fc1-2) mutants suggest that FC1-producing heme is required for initial growth of seedling development. In contrast, weak (fc2-1) and null (fc2-2) mutants of FC2 showed pale green leaves and retarded growth, indicating that FC2producing heme is necessary for chloroplast development. During the initial growth stage, FC2 deficiency caused reduction of plastid cytochromes. In addition, although FC2 deficiency marginally affected the assembly of photosynthetic reaction center complexes, it caused relatively larger but insufficient light-harvesting antenna to reaction centers, resulting in lower efficiency of photosynthesis. In the later vegetative growth, however, fc2-2 recovered photosynthetic growth, showing that FC1-producing heme may complement the FC2 deficiency. On the other hand, reduced level of cytochromes in microsomal fraction was discovered in $f_{c} 1-1$, suggesting that FC1-producing heme is mainly allocated to extraplastidic organelles. Furthermore, the expression of FC1 is induced by the treatment of an elicitor flg22 while that of $F C 2$ was reduced, and $f_{C} 1$ 1 abolished the flg22-dependent induction of FC1 expression and peroxidase activity. Consequently, our results clarified that FC2 produces heme for the photosynthetic machinery in the chloroplast, while FC1 is the housekeeping enzyme providing heme cofactor to the entire cell. In addition, FC1 can partly complement FC2 deficiency and is also involved in defense against stressful conditions.

Keywords: ferrochelatase, heme allocation, cytochromes, photosynthesis, plastid, biotic stress

\section{INTRODUCTION}

Heme (protoheme) is a cofactor responsible for various biological processes including oxygen metabolism, oxygen transfer, electron transfer, and secondary metabolism. Heme also functions as a regulatory molecule (Tanaka et al., 2011).

In plants, heme biosynthesis takes place in plastids by sharing the pathway with chlorophyll biosynthesis until formation of protoporphyrin IX (Masuda et al., 2003; Tanaka et al., 2011). 
Heme is formed directly from protoporphyrin IX by insertion of ferrous $\left(\mathrm{Fe}^{2+}\right)$ ion, whereas chlorophylls are synthesized by several steps after insertion of magnesium ion into protoporphyrin IX. The insertion of $\mathrm{Fe}^{2+}$ into protoporphyrin IX is catalyzed by ferrochelatase. In land plants, two ferrochelatase isoforms of cyanobacterial origin have been identified (Chow et al., 1998; Suzuki et al., 2002), ferrochelatase 1 (FC1) and ferrochelatase 2 (FC2), whose amino acid sequences are about $69 \%$ identical (Chow et al., 1998). A characteristic feature of FC2 is its hydrophobic C-terminal extension, which includes a putative light harvesting chlorophyll $a / b$-binding (LHC) motif (also called CAB domain) (Suzuki et al., 2002). This conserved LHC motif is also present in cyanobacterial ferrochelatase and in FC2-type ferrochelatases from other higher plants. In cyanobacterium Synechocystis sp. PCC 6803, it is reported that the LHC motif is not required for catalytic activity but is essential for dimerization of the ferrochelatase (Sobotka et al., 2010).

These two ferrochelatase isoforms show a clear contrast in gene expression profile such that FC2 is mainly expressed in photosynthetic tissues, whereas FC1 is expressed in all tissues (Chow et al., 1998; Suzuki et al., 2002). Particularly in roots, the FC1 expression is predominant and the FC2 expression is hardly detected, suggesting that FC1 and FC2 have different roles among various tissues. Furthermore, FC1 is strongly upregulated by wounding and oxidative stresses in photosynthetic tissues (Singh et al., 2002; Nagai et al., 2007). Since FC1 is co-induced with genes encoding endoplasmic reticulum (ER)-localized cytochrome P450 family and cytosolic ascorbate peroxidase upon wounding, it is presumed that FC1 supplies extraplastidic heme for defensive functions (Nagai et al., 2007). Actually, Genevestigator analysis showed stress-responsive induction of FC1, in response to drought, wounding, and reactive oxygen species, but not FC2 (Scharfenberg et al., 2015). Meanwhile, FC2 is proposed to be involved in heme production for photosynthetic cytochromes. In fact, gene ontology analysis revealed that genes associated with the term 'photosynthesis' are significantly enriched in the co-expressed genes with FC2. It should be noted that coexpression analysis by Scharfenberg et al. (2015) suggested that genes associated with the term 'defense response to bacteria,' which includes reactions triggered by the pathogens such as Pseudomonas syringae, was identified as other enriched categories of FC2 co-expressed genes.

Mutants of ferrochelatase isoforms have so far been characterized. For FC1, a knock-down mutant $\left(f_{c} 1-1\right)$ was characterized by Nagai et al. (2007) showing reduced heme levels particularly in roots, but photosynthetic parameters such as chlorophyll and carotenoid content, and the efficiency of photosystem II (PSII), were essentially unaffected (Nagai et al., 2007). Woodson et al. (2011) reported that a homozygous null mutant $\left(f_{c} 1-2\right)$ of $F C 1$ could not be recovered from heterozygous parents, suggesting an embryonic-lethal phenotype. Further analysis of this mutant suggests that a second (unlinked) T-DNA insertion may be present that could also cause the lethal phenotype (Scharfenberg et al., 2015). For FC2, weak $(f c 2-1)$ and null ( $\left.f_{c} 2-2\right)$ T-DNA insertion mutants have been isolated (Scharfenberg et al., 2015; Woodson et al., 2015). Phenotypic analysis of $f c 2-1$ showed that the mutant seedlings are abnormally small with pale green rosette leaves, low in chlorophylls, carotenoids and several photosynthetic proteins, and impaired photosynthetic performance (Scharfenberg et al., 2015; Woodson et al., 2015). Moreover, it was found that the lack of FC2 resulted in a fluorescent ( flu)-like phenotype (Scharfenberg et al., 2015; Woodson et al., 2015). In the flu mutant, the photosensitizer protochlorophyllide accumulates in the dark (Meskauskiene et al., 2001). Consequently, exposure of the flu mutant to light generates singlet oxygen $\left({ }^{1} \mathrm{O}_{2}\right)$ and $\mathrm{flu}$ seedlings bleach and die. Although accumulating species of tetrapyrroles are different between Scharfenberg et al. (2015) (i.e., protochlorophyllide accumulation) and Woodson et al. (2015) (i.e., protoporphyrin IX accumulation), $f c 2-1$ and $f c 2-2$ were found to exhibit $f l u$-like phenotype when they are grown in the dark unlike $f c 1-1$ mutant.

In addition to the differences in gene expression, a distinct involvement of FC1- and FC2-derived heme in retrograde plastid signaling has been proposed (Woodson et al., 2011). Woodson et al. (2011) performed a gain-of-function genetic screening of genomes-uncoupled (gun) mutants using activationtagged lines of Arabidopsis, and subsequently discovered that overexpression of FC1 restores nuclear-encoded photosynthesisassociated gene expression even when chloroplast development is blocked. These data suggest that increased flux through the FC1-producing heme may act as a signaling molecule that control photosynthesis-associated nuclear genes as retrograde signal. Although FC1 and FC2 colocalized to the same plastids and utilized the same biosynthetic pathway, overexpression of FC2 failed to derepress photosynthesis gene expression (Woodson et al., 2011). Furthermore, genetic complementation of $f c 2-1$ showed that expression of FC1 could not prevent the accumulation of protoporphyrin IX, but restored wildtype levels of heme and chlorophyll in constant light and protochlorophyllide in the dark (Woodson et al., 2015). These results suggest that although FC1 and FC2 are colocalized in plastids and function for heme biosynthesis, FC2-derived heme is allocated differently from FC1-derived heme that can be transferred to extraplastidic locations and function in stressresponses or retrograde signaling. However, the allocation of heme produced by each ferrochelatase isoforms in plant cells is not well understood.

In this study, we re-examined T-DNA insertional Arabidopsis mutants deficient in ferrochelatase isoforms. By further analysis of these mutants, we showed that FC1 and FC2 have distinct physiological functions for developmental growth. Furthermore, these isoforms are distinctly involved in heme allocation inside and outside plastids. Thus, our data demonstrate that the allocation of heme is differentially regulated by FC1 and FC2 in plant cells.

\section{MATERIALS AND METHODS}

\section{Plant Materials and Growth Conditions}

The Arabidopsis T-DNA insertional mutants of ferrochelatase isoforms, $f c 1-1$ (SALK_150001), $f c 1-2$ (GK_110D_02), $f c 2-1$ (GK_766_H08), and $f c 2-2$ (SAIL_20_C06), are Columbia ecotype 
and obtained from ABRC stock center. Seeds were surfacesterilized before sowing on solidified Murashige and Skoog medium (Murashige and Skoog, 1962) containing 1\% (w/v) sucrose and $1 \%(\mathrm{w} / \mathrm{v})$ gelrite (Duchefa) at $22^{\circ} \mathrm{C}$ under continuous white light (35-45 $\mu \mathrm{mol}$ photons $\left.\mathrm{m}^{-2} \mathrm{~s}^{-1}\right)$. All data represent three biological replicate experiments. For flg22 (Sawady Technology, GenScript) treatment, sterilized seeds were cultured in $15 \mathrm{ml}$ of $0.1 \mathrm{x}$ MS liquid medium containing $0.1 \%$ sucrose (w/v) and buffered to $\mathrm{pH} 5.7$ with $0.05 \mathrm{~g} \mathrm{l}^{-1} \mathrm{MES}$ at $110 \mathrm{rpm}$, $22^{\circ} \mathrm{C}, 60 \mu \mathrm{mol}$ photons $\mathrm{m}^{-2} \mathrm{~s}^{-1}$ on a gyratory shaker (ShakeLR, Taitec) for indicated days, added indicated concentration of flg22, and cultured for indicated periods.

\section{Genotyping and Quantitative Reverse Transcriptase-Polymerase Chain Reaction (qRT-PCR) Analysis}

Genomic DNA and total RNA were isolated using Nucleon Phytopure Plant Extraction Kit (Thermo Scientific) and RNeasy Mini Kit (Qiagen), respectively, and nucleotide concentration was determined using NanoDrop (ND-1000; Thermo Scientific) spectrophotometer. For genotyping, isolated DNA was subjected to PCR amplification with specific primers and obtained products were electrophoresed in $1 \%$ agarose gel. For qRT-PCR, $1 \mu \mathrm{g}$ of total RNA was reverse transcribed using PrimeScript II 1st strand cDNA Synthesis Kit (Takara). qRT-PCR was performed with specific primer sets using Thunderbird SYBR qPCR Mix (Toyobo) on a Mini Opticon Real-Time PCR System (Bio-Rad). ACTIN8 was used to compute the relative transcript abundance. All primers used are listed in Supplementary Table 1.

\section{Measurement of Photosynthetic Pigments}

Chlorophyll content was measured as described (Arnon, 1949; Melis et al., 1987). Heme contents were measured by highly sensitive assay using horseradish peroxidase as described (Masuda and Takahashi, 2006; Takahashi and Masuda, 2009; Espinas et al., 2012).

\section{GUS Staining}

By using FC1 promoter fused $\beta$-glucuronidase (GUS) transgenic line (FC1pro::GUS), histochemical analyses for GUS expression were carried out as described (Nagai et al., 2007).

\section{Protein Extraction and Western Blot Analysis}

Total and separated proteins were subjected to SDS-PAGE. Soluble (S) and membrane fractions (M) were prepared by centrifugation of plant homogenates for $10 \mathrm{~min}$ at $10,000 \mathrm{~g}$ under $4^{\circ} \mathrm{C}$. Protein contents were determined using $R C D C$ Protein Assay (Bio-Rad). In each lane, $40 \mu \mathrm{g}$ of proteins were loaded. After SDS-PAGE in $12.5 \%$ polyacrylamide gels, proteins were electrophoretically transferred to a nitrocellulose membrane (Hybond- $\mathrm{N}^{+}$, Thermo Scientific) and subsequently exposed to antibodies tested. The blot was then incubated with anti-rabbit immunoglobulin G conjugated to horseradish peroxidase, following which the proteins were detected using chemiluminescence reagent (Millipore). All antibodies used are listed in Supplementary Table 2.

\section{Blue Native PAGE}

For Blue Native PAGE, thylakoid membranes were isolated from the leaves of 2-week-old plants as described (Takahashi et al., 2014). Solubilized membrane proteins containing $5 \mu \mathrm{g}$ chlorophyll were then separated by $5-14 \%$ acrylamide gradient gels according to the method of Wittig et al. (2006).

\section{Chlorophyll Fluorescence Measurement}

Photochemical efficiency analysis was performed using a pulse amplitude modification (PAM) fluorometer (Junior-PAM, Walz) from the leaves of 2-week-old plants as described (Kobayashi et al., 2013). From the obtained fluorescence yields $\left(F_{\mathrm{o}}, F_{\mathrm{m}}, F_{\mathrm{o}}^{\prime}\right.$, and $F_{\mathrm{m}}^{\prime}$ ), photosynthetic parameters were calculated according to the previous equations (van Kooten and Snel, 1990; Maxwell and Johnson, 2000). The $\Phi_{\mathrm{NPQ}}$ and $\Phi_{\mathrm{NO}}$ were determined according to the method of Kramer et al. (2004).

\section{Heme Staining}

Microsomal light membrane (LM) fraction was extracted by layering membrane fractions on $0.81 \mathrm{M}$ sucrose, followed by ultracentrifugation (Optima, Beckman, Inc.) for $1 \mathrm{~h}$ at 100,000 $\mathrm{g}$. This fraction was solubilized in $62.5 \mathrm{mM}$ Tris- $\mathrm{HCl}, \mathrm{pH} 6.8,4 \%$ SDS, $20 \%$ glycerol, and $0.2 \%$ bromothymol blue. After separation by non-reducing SDS-PAGE, gels were stained with $6.3 \mathrm{mM}$ $3,3^{\prime}, 5,5^{\prime}$-tetramethylbenzidine (Nacalai Tesque) in methanol and $0.25 \mathrm{M}$ sodium acetate in 3:7 (v/v) ratio, respectively. Hydrogen peroxide $\left(\mathrm{H}_{2} \mathrm{O}_{2}\right)$ was then added to a final concentration of $30 \mathrm{mM}$. The visible staining was scanned using a flatbed scanner.

\section{Peroxidase Assay}

Plants were grown on soil for 27 days as described (Nozaki et al., 2012). Excised rosette leaves were soaked and vacuum infiltrated with a solution of $0.5 \mathrm{\mu g} \mathrm{ml}^{-1}$ flg22 containing $0.001 \%$ Triton X100 , incubated in the solution for $24 \mathrm{~h}$ at $22^{\circ} \mathrm{C}$ in darkness and stored at $-60^{\circ} \mathrm{C}$. Extraction of peroxidase activities was carried out as described (Sato et al., 2011). Briefly, the stored leaves were homogenized in $50 \mathrm{mM}$ Tris-HC1 buffer ( $\mathrm{pH}$ 7.2) using a plastic pestle and then using an ultrasonic disruptor (UD-200, Tomy Seiko). The homogenate was centrifuged at $1,500 \mathrm{~g}$ for $5 \mathrm{~min}$. The supernatant was re-centrifuged at $15,000 \mathrm{~g}$ for $20 \mathrm{~min}$ and used for assay of soluble peroxidase activities. Ionically bound peroxidase activities were extracted with $1 \mathrm{M} \mathrm{NaCl}, 50 \mathrm{mM}$ Tris$\mathrm{HCl}$ buffer ( $\mathrm{pH}$ 7.2) from the pellet of cell walls. Peroxidase activities were measured as described (Sato et al., 1993). The final reaction mixture $(100 \mu \mathrm{l})$ contained $5 \mu \mathrm{l}$ of enzyme preparation, $13 \mathrm{mM}$ guaiacol, $15 \mathrm{mM} \mathrm{CaCl}_{2}, 5 \mathrm{mM} \mathrm{H}_{2} \mathrm{O}_{2}$, and $40 \mathrm{mM}$ Tris$\mathrm{HCl}$ ( $\mathrm{pH}$ 7.2). The sum of soluble and ionically bound peroxidase activities was regarded as total peroxidase activity.

\section{Lignin Assay}

For disruption, 5-6 seedlings frozen in liquid nitrogen were disrupted with a bead-type cell disrupter (MS-100, Tomy Seiko). Lignin assay was carried out as described (Schenke et al., 
2011). For conversion to relative units based on absorbance the following relation was used: $100 \mu \mathrm{g}$ lignin in $1 \mathrm{ml}$ produce an $A_{280}$ of 0.60 in a 1-cm cell according to Müsel et al. (1997).

\section{Statistics}

All statistics were performed using two-tailed Student's $t$-test. Asterisk indicate significant difference in $P<0.05$ to wild-type control.

\section{RESULTS}

\section{Analysis of Mutants of Ferrochelatase Isoforms}

In this study, to analyze physiological functions of ferrochelatase isoforms, we firstly re-examined the T-DNA insertion mutants of ferrochelatase isoforms in Arabidopsis thaliana.
For FC1, $f c 1-1$ (SALK_150001) and fc1-2 (GK_110D_02) have so far been characterized (Nagai et al., 2007; Scharfenberg et al., 2015; Woodson et al., 2015). $f c 1-1$ possesses a single copy of T-DNA in the $5^{\prime}$-untranslational region of FC1, while in $f c 1-2$, T-DNA insertion was localized in the third exon of FC1 (Figure 1A). As reported previously (Nagai et al., 2007), $f(c 1-1$ was almost comparable to wild-type phenotype (Figure 2; Supplementary Figure S1) and lacked stress-induced FC1 expression (Figure 8A). qRT-PCR analysis showed about $80 \%$ reduction in the transcript levels of $F C 1$ in $f c 1-1$ even under normal growth condition (Figure 1B). Meanwhile, it was reported that a homozygous null $f c 1-2$ mutant is likely to be embryonic lethal (Woodson et al., 2011). Subsequently, it was suggested that the presence of a second unlinked T-DNA insertion is involved in the lethal phenotype of this line (Scharfenberg et al., 2015). Here, we found that some of homozygous $f c 1-2$ seeds were able to germinate, emerging as a
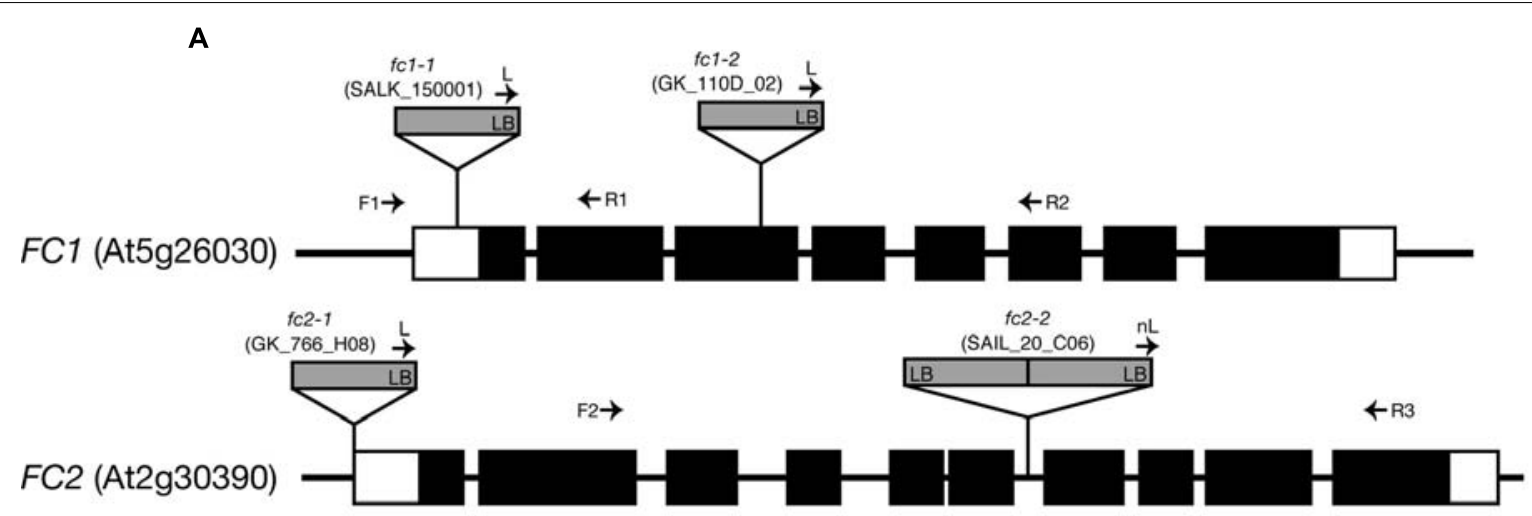

B

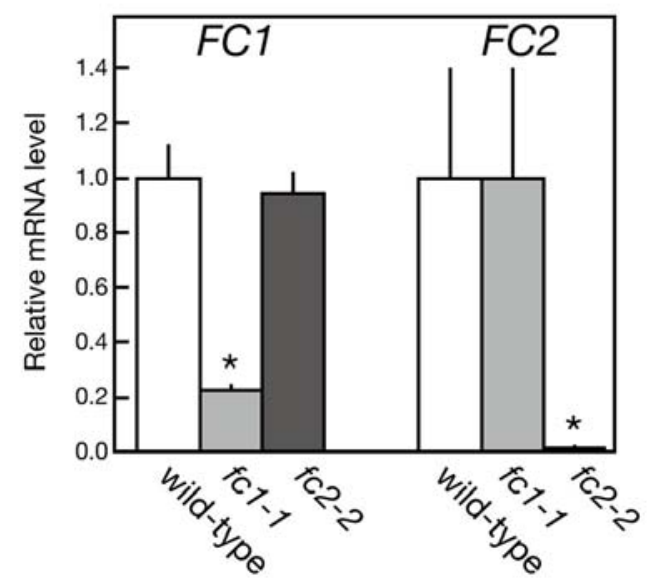

C

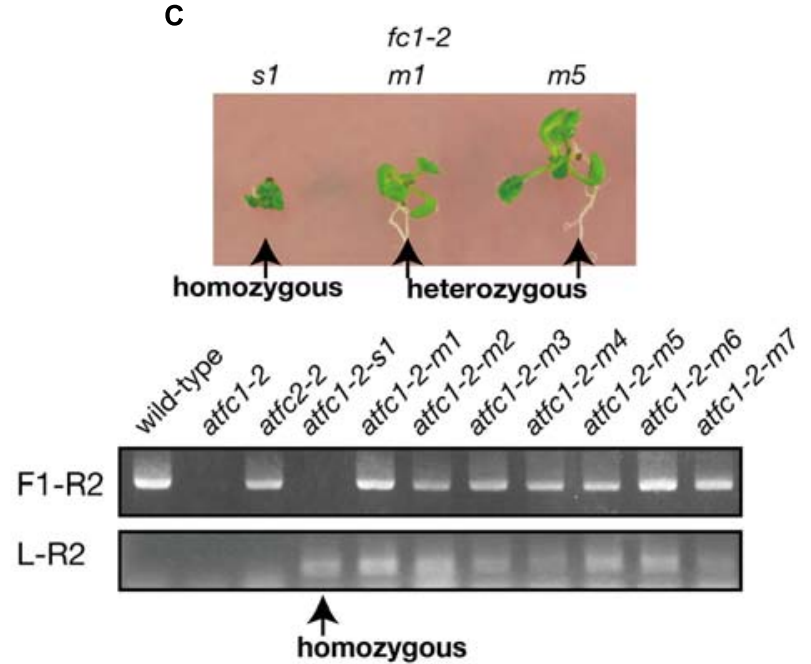

FIGURE 1 | Characterization of T-DNA insertional mutants of ferrochelatase isoforms. (A) Schematic representation and T-DNA tagging of FC1 (At5g26030) and FC2 (At2g30390) loci. Exons (black boxes) and untranslated regions (white boxes) are shown. Location and orientation of T-DNA insertions in each line are indicated. Arrows represent the primers used for genotyping of FC1 and FC2 (see Supplementary Table 1). (B) qRT-PCR analysis of FC1 and FC2 mRNA transcripts extracted from 7-day-old wild type, fc1-1, and fc2-2 seedlings. Values are presented as the fold difference from the wild-type after normalizing to the control gene ACTIN8. Bars indicate standard error of the mean (SEM) from three independent experiments. Asterisks indicate a significant difference from the wild-type $\left({ }^{*} P<0.05\right.$, Student $t$-test). (C) Phenotypic and genomic analysis of $f c 1-2$. (Upper) Photographs of small ( $\left.s 1\right)$ and middle ( $m 1$ and $\left.m 5\right)$ size seedlings germinated from heterozygous fc1-2 seeds. (Lower) PCR-based genomic analysis of these seedlings, showing small size seedling (s1) is actually homozygous fc1-2 seedling. 
very small seedling (Figure 1C). The germination rate was quite low $(<1 \%)$, which is far lower than theoretical segregation rate of homozygous $f c 1-2$ (25\%). PCR-based genome analysis confirmed that the small seedlings are actually homozygous $f c 1-2$ mutant (Figure 1C lower panel). The homozygous $f c 1-2$ was green but its growth was severely retarded and it died during initial seedling stage, suggesting that homozygous $f_{c} 1-2$ can be occasionally successful for embryogenesis but seedlings are arrested during further development. Because $f c 1-2$ seedling was too small and its occurrence was too rare to be assayed, it was excluded from further analysis.

For FC2, fc2-1 (GK_766_H08) and fc2-2 (SAIL_20_C06) have so far been characterized (Scharfenberg et al., 2015; Woodson et al., 2015). In $f c 2-1$, T-DNA insertion is located in the $5^{\prime}$-untranslated region, while in $f_{c} 2-2$, a single copy of T-DNA in tandem is inserted between exons 6 and 7 of FC2 (Figure 1A). Here we analyzed $f_{c} 2-2$ that shows more severe phenotype than $f_{c} 2-1$ (Scharfenberg et al., 2015). After obtaining homozygous $f c 2-2$ mutant, we observed the deficiency of the FC2 mRNA (Figure 1B) in the mutant. Consistent with previous reports (Scharfenberg et al., 2015; Woodson et al., 2015), fc2-2 showed pale-green phenotype with substantial decrease in their weight and chlorophyll content during 1 week after germination (Figures $\mathbf{2 A}-\mathbf{C}$ ). In $f c 2-2$, the chlorophyll $a / b$ ratio was lower than that of wild-type, suggesting relatively high abundance of LHC proteins to reaction centers (Figure 2C). During further development for 1 more week, $f c 2-2$ became greener (Figures 2B,C) and finally it behaved like wild-type and became fertile (Supplementary Figure S1D). The levels of total heme per seedling in $f_{c} 2-2$, however, remained almost $30 \%$ of wild-type during 2 weeks after germination (Figure 2D). We could not detect the reduction of heme in $f c 1-1$ probably because the effect of FC1 deficiency in heme production is rather limited in roots and the difference of heme was not detectable in the seedling level (Nagai et al., 2007). The level of reduction of total heme in $f_{c} 2-2$ were similar to that reported by Woodson et al. (2015), but more severe than those observed by Scharfenberg et al. (2015). It should be noted that consistent with previous
A

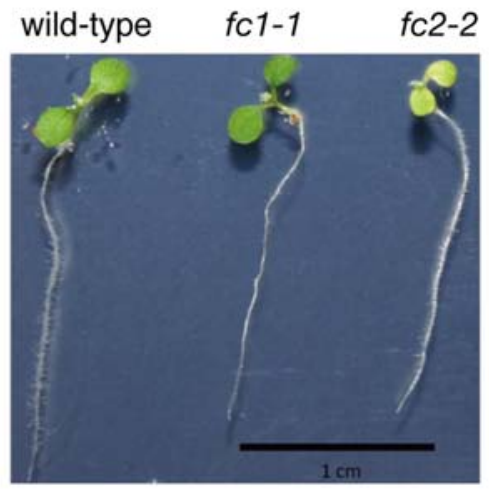

C

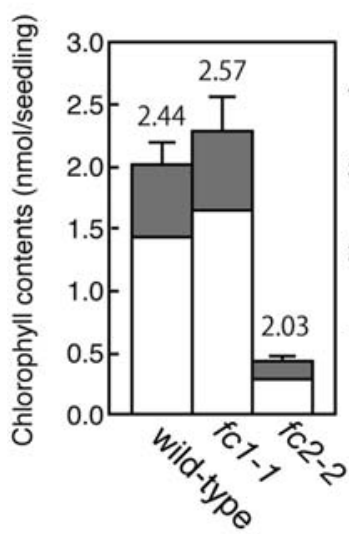

7-day-old

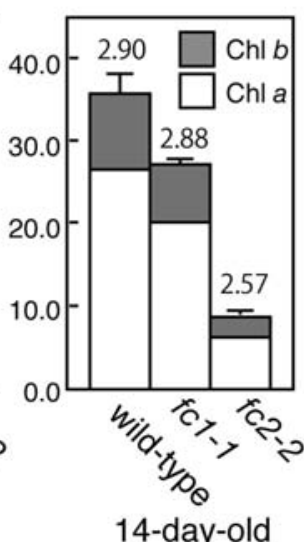

14-day-old
B
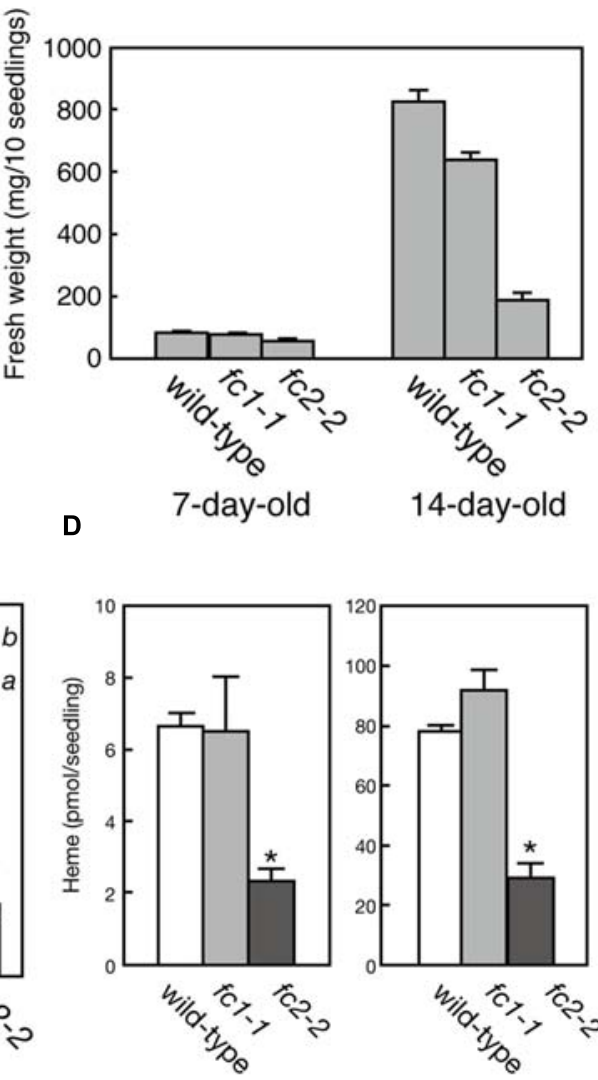

7-day-old

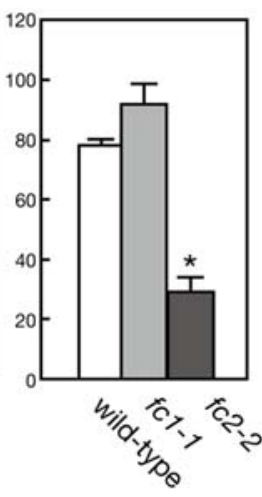

14-day-old

FIGURE 2 | Phenotypic characterization of fc1-1 and fc2-2. (A) Photographs of 7-day-old wild-type, homozygous fC1-1, and homozygous fC2-2 seedlings. Seedlings of $f_{c} 2-2$ show pale-green phenotype. (B) Fresh weights of wild-type, $f_{c} 1-1$, and fc2-2 of 7-and 14-day-old seedlings. (C) Chlorophyll contents of wild-type, $f_{C} 1-1$, and $f_{C} 2-2$ of 7 - and 14-day-old seedlings. Chlorophyll a (white bars) and $b$ (gray bars) contents are indicated. Numbers on the bars are chlorophyll a/b ratio. (D) Total endogenous heme contents in 7- and 14-day-old seedlings in wild-type, fC1-1, and fc2-2. Asterisks indicate a significant difference from the wild-type $\left({ }^{*} P<0.05\right.$, Student $t$-test). In all figures, bars indicate standard error of the mean (SEM) from three independent experiments. 
studies (Scharfenberg et al., 2015; Woodson et al., 2015) $f c 2-2$ showed $f l u$ phenotype when they were grown in the dark (data not shown).

We also produced $f_{c} 1-1 f_{c} 2-2$ double mutant by crossing (Supplementary Figures S1A,B). The obtained homozygous $f_{c} 1-1 f_{c} 2-2$ double mutant showed more severe phenotype than parental lines with much reduced size and paler cotyledons than those of $f c 2-2$ (Supplementary Figures S1B,C). On MS solidified medium or soil, the homozygous $f_{c} 1-1 \quad f_{c} 2-2$ double mutant seedlings stopped their growth before or soon after bolting and died, so they are infertile (Supplementary Figures S1D,E). These results show that FC1 and FC2 have distinct physiological functions for developmental growth. Considering severe phenotype of $f c 1-2, F C 1$ supplies heme essential for housekeeping function including embryogenesis. FC2-produced heme seems important for chloroplast development but not essential for seedling development. In the later developmental stage, it is likely that the deficiency of FC2 can be complemented by the function of FC1, but not vice versa.

\section{Histochemical Analysis of FC1 Expression during Development}

To observe the expression of FC1 during development, we performed histochemical analysis of FC1 by using FC1 promoter fused GUS line (FC1pro::GUS) (Nagai et al., 2007) (Figure 3). In young 2-day-old seedlings (Figures 3A,B), FC1 expression was mainly observed in roots, with prominent staining in regions of vein and root cap, but not elongation zone. In cotyledons, faint staining was observed in vein regions. In mature seedlings (2- and 3-week-old), FC1 expression was also mainly observed in roots (Figures 3C,D). High expression of FC1 was detected in primordial tissues of leaves and stipules in 2-week-old seedlings and bolting shoot in 3-weekold seedlings (Figures 3C-E,H). Meanwhile, in developed leaves, faint expression of FC1 was observed, and its expression was much reduced in 3-week-old seedlings when compared to 2-week-old seedlings (Figures 3C,D). In roots of matured plants, the expression of FC1 was mainly observed in vein regions but not in root caps (Figures 3F,G). These results show that in addition to the housekeeping expression in roots, FC1 is highly expressed in primordial newly emerging tissues, such as new leaves, stipules, and bolting stems, suggesting its important role for the activity of the apical shoot meristem (Figures 3C-E,H). Since FC2 is preferentially expressed in photosynthetic tissues (Suzuki et al., 2002), and its expression is highly analogous to other photosynthetic genes (Scharfenberg et al., 2015), histochemical analysis of FC2 expression was not performed in this study.

\section{Effect of FC2 Deficiency on the Assembly of Photosynthetic Machineries}

We then performed the immunoblot analysis on $f c 1-1$ and $f c 2-2$ mutants. Each sample was loaded on the same amount of protein basis. As shown in Figure 4, FC2 protein was undetectable in $f c 2-2$, confirming null mutation of FC2. Cytochromes $b_{6} f$ is present in chloroplast and functions in the photosystem electron transport chain. As shown by immunoblot analysis in Figure 4, the amount of cytochrome $f$ (PetA) was pronouncedly reduced in $f c 2-2$, indicating that formation of cytochrome $b_{6} f$ in chloroplasts requires heme supply from FC2-dependent pathway. We noticed that the level of $\alpha$ subunit of cytochrome $b_{559}$ (PsbE) was also severely reduced in $f c 2-2$ (Figure 4B). Meanwhile, the levels of both plastid cytochromes were almost unaffected in $f_{c} 1-1$. These results suggest that heme allocated to chloroplasts is mainly attributed by FC2-dependent pathway. On the other hand, the contribution of FC1 to heme supply to chloroplasts may be limited and therefore FC1 cannot complement the chloroplast defects in $f c 2-2$ during the initial stage of development. We also found that the level of HEMA1, a predominant isoform of glutamyl-tRNA reductase, is certainly accumulated in $f_{c} 2-2$ (Figure 4B). HEMA1 is a rate-limiting enzyme of total flow of tetrapyrrole biosynthesis (Tanaka et al., 2011), so the supply of porphyrin intermediates may be increased in $f_{c} 2-2$.

We also determined the levels of photosynthetic proteins (Figure 4B). In $f_{c} 2-2$, similar to the reduction of cytochrome $b_{6} f$, reduction of other photosynthetic proteins was observed. It seems that the FC2 deficiency globally affected the amounts

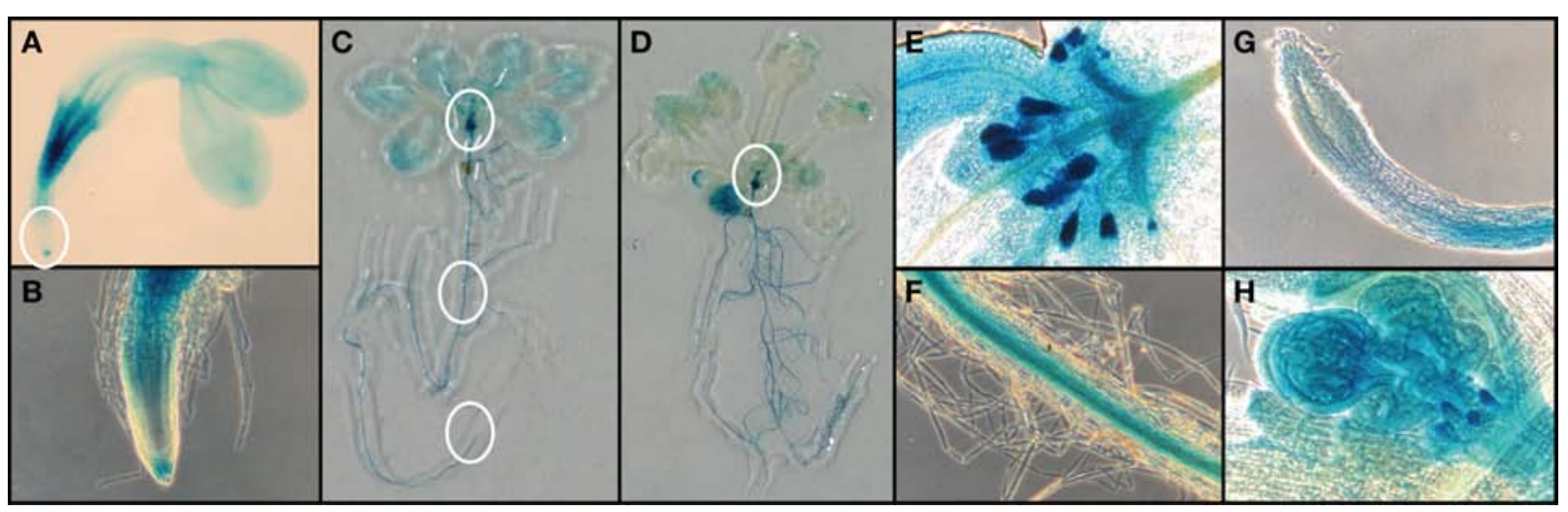

FIGURE 3 | Histochemical analysis of FC1pro::GUS line. (A) FC1-dependent GUS activities in 2-day-old seedlings. (B) Zoomed image of regions of root cap and elongation zone as indicated by a white circle in (A). Representative images of (C) 14-d-old seedling and (D) 21-day-old seedling. Zoomed image of regions of (E) primordial leaves, (F) root veins, and (G) veins as indicated by white circles in (C). (H) Zoomed image of primordial bolting stem as indicated by a white circle in (D). 


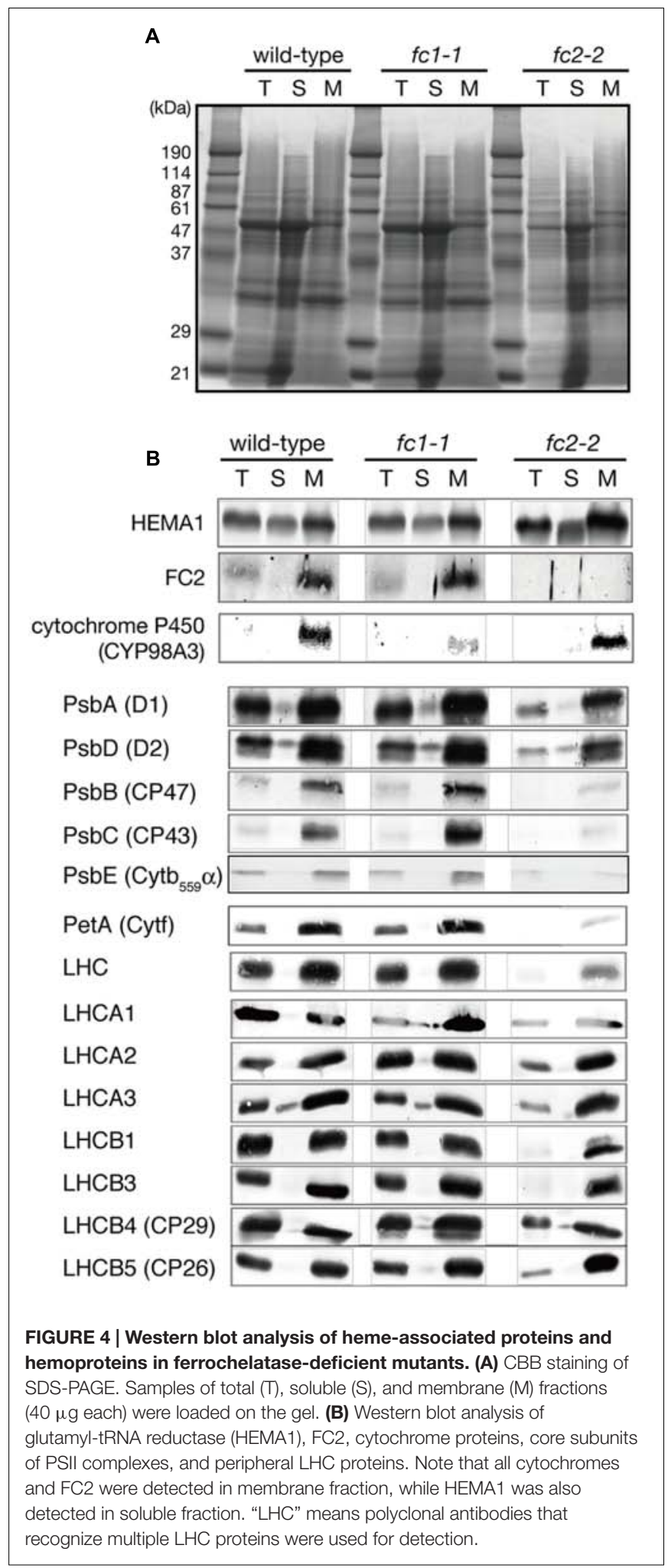

of photosynthetic machinery, such as reaction center proteins as well as LHC antenna proteins. Although the levels of reduction were varied among proteins, the levels of reduction of photosynthetic proteins were less pronounced than those of

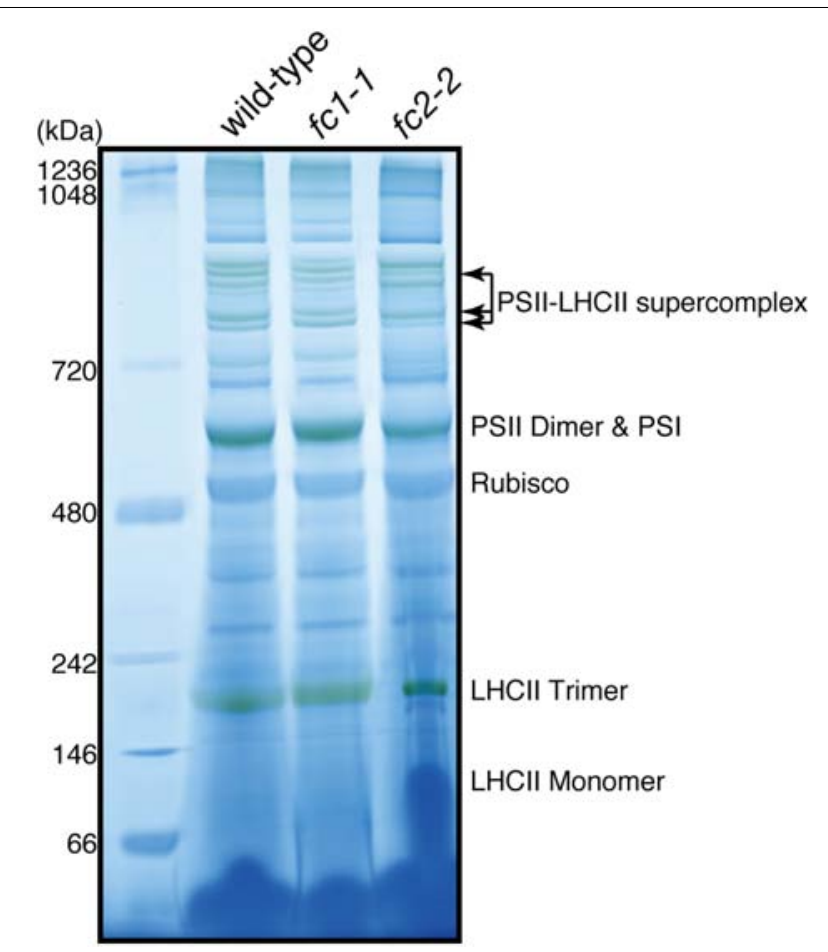

FIGURE 5 | Blue-native PAGE analysis of photosystem complexes in wild-type and ferrochelatase deficient mutants. Thylakoid membrane fractions obtained from 14-day-old seedlings are solubilized. Proteins containing $5 \mu \mathrm{g}$ chlorophyll were loaded to $4-14 \%$ linear gradient gel. Molecular size markers are indicated on the left.

cytochromes $f$ and PsbE. Exceptions are $\mathrm{CP} 43$ and $\mathrm{CP} 47$ proteins showing much severe reduction than any other photosynthetic proteins. We then performed blue native gel analysis of membrane proteins loaded on the same amount of chlorophyll basis (Figure 5). When compared to wild-type and $f c 1-1, f c 2-2$ deficiency caused slightly different band profile of PSII-LHCII supercomplex, probably because of different composition of PSII subunits (Figure 4B). Comparing to reaction center complexes, relative band intensities of LHCII trimer and monomer bands were higher in $f c 2-2$. Considering a lower chlorophyll $a / b$ ratio, relatively higher amounts of LHC proteins to reaction center proteins may be present in $f c 2-2$. It is likely that heme deficiency in $f c 2-2$ mainly affected the assembly and connection of LHC antenna to reaction center complexes, but not the assembly of PSI and PSII reaction center complexes.

To further examine the impact of FC1 or FC2 deficiency on the photosynthetic electron flow, we analyzed their chlorophyll fluorescence using PAM techniques (Maxwell and Johnson, 2000). The light-response curves of Chl fluorescence from PSII showed that the effective photochemical quantum yield of PSII $\left(\Phi_{\text {II }}\right)$ in $f c 2-2$ was lower than those in wild-type and fc1-1 under low to high photosynthetically active radiation (PAR) (Figure 6A). This trend is consistent with previous observation using $f_{c 2}-1$ (Scharfenberg et al., 2015), but much more pronounced in $f c 2-2$. In Figure $\mathbf{6 A}$, the $\Phi_{\text {II }}$ values in darkness $\left(0 \mu \mathrm{mol}\right.$ photon $\left.\mathrm{m}^{-2} \mathrm{~s}^{-1} \mathrm{PAR}\right)$ represent the maximum 


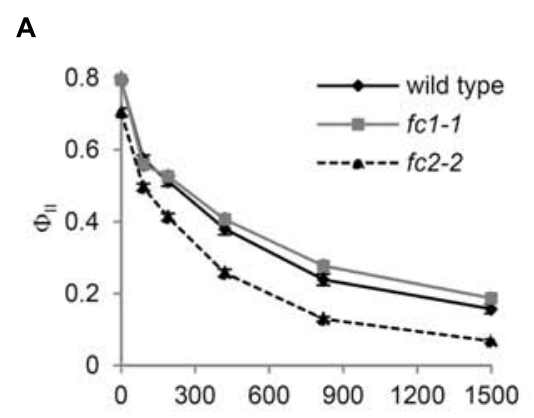

D

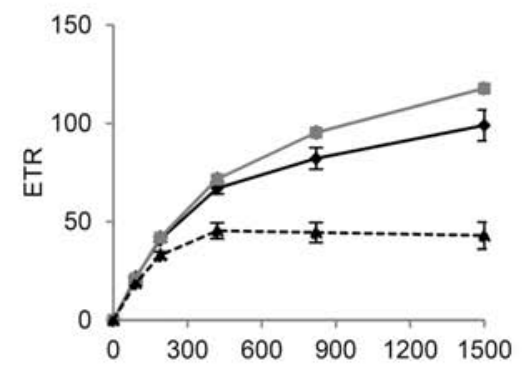

B

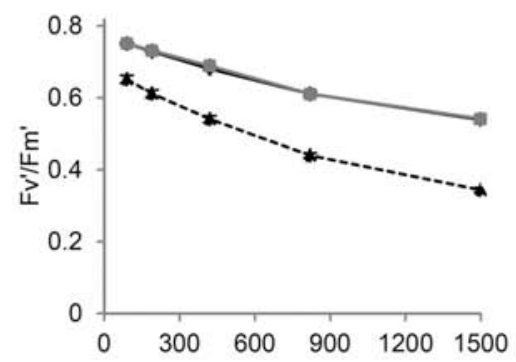

E

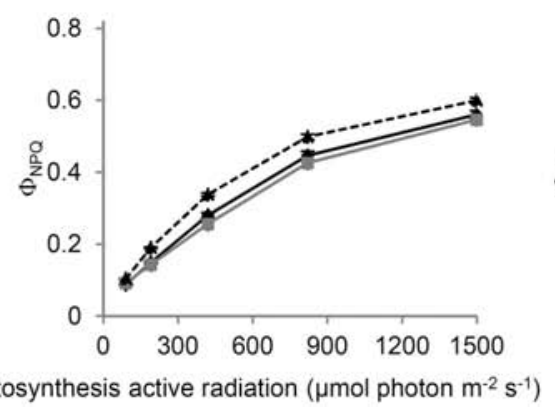

C

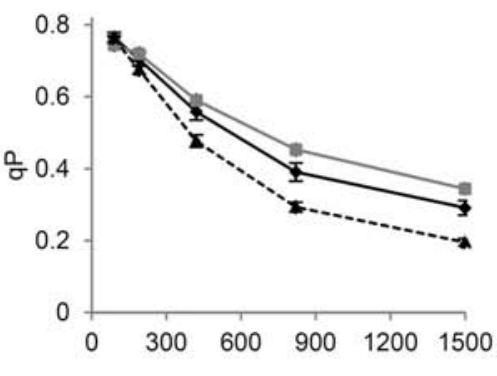

$\mathbf{F}$

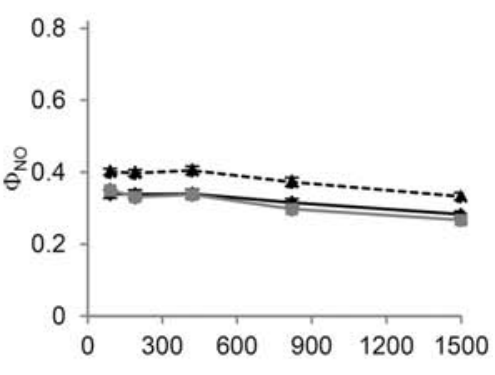

FIGURE 6 | Chlorophyll fluorescence parameters of wild-type and ferrochelatase deficient mutants. Light response curves of (A) $\Phi_{\|}$, (B) $F_{v}{ }^{\prime} / F_{m}{ }^{\prime},($ C) $q P$, (D) ETR, (E) $\Phi_{\mathrm{NPQ}}$, and (F) $\Phi_{\mathrm{NO}}$ are shown. $\Phi_{\|}$, PSIl quantum yield/operating quantum efficiency of PSIl photochemistry; $F_{\mathrm{v}}{ }^{\prime} / F_{\mathrm{m}^{\prime}}$, efficiency of open PSII reaction centers; qP, fractions of PSIl centers in open states based on puddle model for the photosynthetic unit; ETR, electron transfer rate in PSIl; $\Phi_{N P Q}$, quantum yield of light-induced non-photochemical quenching (NPQ)/NPQ efficiency; $\Phi_{N O}$, non-regulated energy dissipation. PAR means photosynthetic active radiation. Bar indicates SEM from five independent experiments for wild type and fc2-2. In addition, two biological replicates of the $f_{c} 1-1$ data were included as comparison.

quantum yield of PSII $\left(F_{\mathrm{v}} / F_{\mathrm{m}}\right)$, and that was decreased to 0.70 in $f c 2-2$ compared with 0.79 in wild type. Consistently, the maximum quantum yield of open (oxidized) PSII under light $\left(F_{\mathrm{v}}^{\prime} / F_{\mathrm{m}}^{\prime}\right)$ was lower in $f_{c} 2-2$ than the wild type and $f_{c} 1-1$ under all ranges of light intensity (Figure 6B). These data suggest that the intrinsic photochemical activity of PSII is impaired in $f_{c} 2-2$. Moreover, the fraction of open PSII represented by coefficient of the photochemical quenching (qP) was decreased in $f c 2-2$ compared to wild-type and $f c 1-1$ (Figure 6C). Electron transport rate (ETR) of PSII calculated from $\Phi_{\text {II }}$ was substantially lower in $f_{c} 2-2$ with reaching to a plateau at lower PAR than the wild type and $f c 1-1$ (Figure 6D). Thus, decreased openness of PSII with decreased intrinsic photochemical efficiency resulted in retarded photosynthetic electron transport in $f c 2-2$.

Absorbed light energy by LHCII-PSII can be divided into $\Phi_{\mathrm{II}}$, quantum yield of light-induced non-photochemical quenching $\left(\Phi_{\mathrm{NPQ}}\right)$ and quantum yield of non-regulated energy dissipation $\left(\Phi_{\mathrm{NO}}\right)$ (Kramer et al., 2004). In $f_{c} 2-2$, decreased $\Phi_{\mathrm{II}}$ was reflected in higher values of both $\Phi_{\mathrm{NPQ}}$ (Figure 6E) and $\Phi_{\mathrm{NO}}$ (Figure 6F). These results suggest that in $f c 2-2$, the absorbed light energy is not efficiently transferred from LHC antenna to PSII reaction centers and dissipated as heat or fluorescence.

\section{Impact of FC1 Or FC2 Deficiency on Hemoproteins}

Our previous study showed that FC1 was co-expressed with wounding-inducible cytochrome P450 family (Nagai et al., 2007), implying a relationship between FC1-dependent heme pathway and cytochrome $\mathrm{P} 450$ proteins. To confirm whether the level of cytochrome $\mathrm{P} 450$ is affected in $f c 1-1$ or not, we examined the abundance of a representative cytochrome P450 (CYP98A3) by immunoblot analysis (Figure 4B). CYP98A3, whose mRNA can be detected in all organs of Arabidopsis, is involved in the conversion of $p$-coumaroyl quinate into chlorogenic acid, and also is expected to catalyze the meta-hydroxylation step for the formation of lignin monomers (Bak et al., 2011). The level of CYP98A3 was actually reduced in $f c 1-1$, while it remained to the wild-type level in $f c 2-2$ (Figure 4B), showing that the FC1dependent pathway and not FC2 is required for the accumulation of ER-localized CYP98A3.

The decrease in CYP98A3 in $f c 1-1$ suggests an essential role of FC1 in heme supply to cytochrome P450 proteins. Since almost all plant cytochrome $\mathrm{P} 450$ proteins are known to bind the cytoplasmic surface of ER (Bak et al., 2011), we isolated the ERenriched microsomal LM fraction from seedlings and performed heme staining (Figure 7A). In this experiment, hemoproteins in non-reducing gel were detected by peroxidase activity of heme. As shown in Figure 7A, two major bands (220-240 and $120 \mathrm{kDa}$ ) were detected, which are presumably representing cytochrome $\mathrm{P} 450$ proteins as oligomeric forms. Interestingly, $f c 1-1$ showed less staining of these bands, while $f_{c} 2-2$ showed more intense staining than wild-type. To test whether the levels of staining are actually related to the extractable heme levels, we performed heme determination in LM fraction with highly 


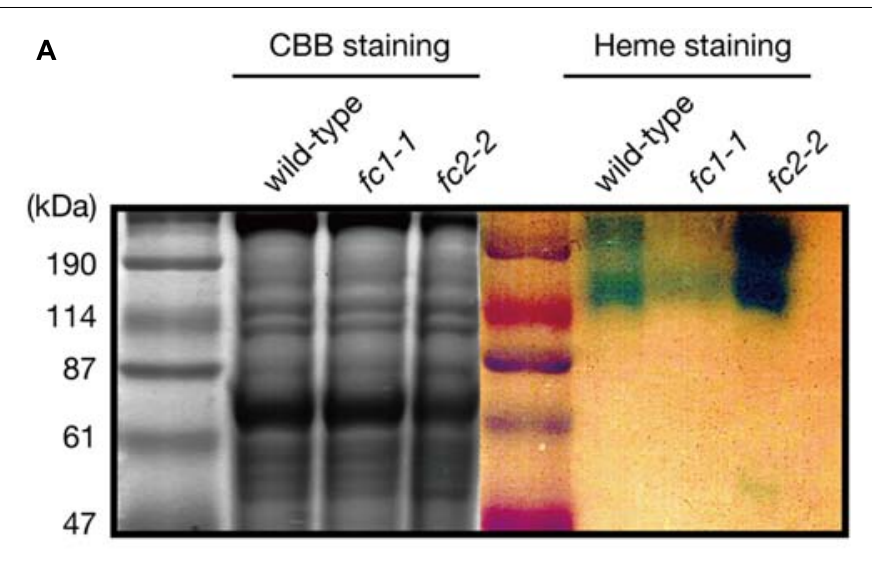

\section{B}

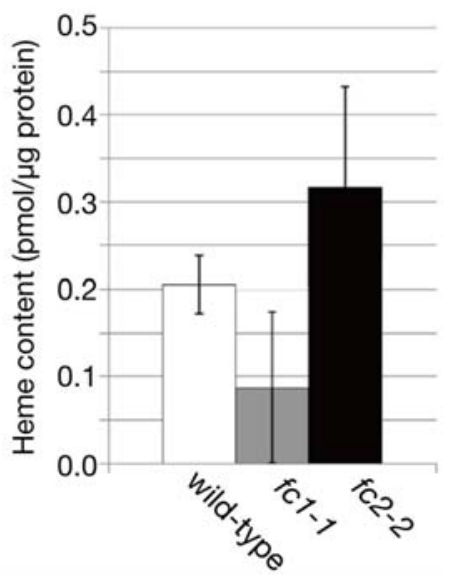

FIGURE 7 | Heme staining of ER-enriched microsomal light membrane (LM) fraction. (A) CBB and heme staining of the LM fraction (30 $\mu$ g/well) of wild type $f_{C} 1-1$, and $f_{C} 2-2$. Blue staining represents the heme-dependent peroxidase activity. (B) Heme levels in LM fractions were determined by highly sensitive heme assay. Bars indicate SEM from three independent experiments.

sensitive heme assay (Takahashi and Masuda, 2009; Espinas et al., 2012) (Figure 7B). Because of very low heme levels in each fraction, the obtained data were fluctuated. Although heme levels in each fraction showed trends similar to heme staining data, they were not statistically validated. Considering the total heme level was unchanged in $f_{c} 1-1$ and decreased in $f_{c} 2-2$, heme produced by FC1 and FC2 may allocate differentially to various organelles. Our data suggest that FC1 is involved in supply of extraplastidic heme to LM fraction, while FC2 is not. Furthermore, under FC2 deficiency, such extraplastidic heme supply is likely to be upregulated. It is interesting to note that, in $f c 2-2$, the abundance of the HEMA1 protein was certainly increased (Figure 4B) whereas the expression of FC1 was not upregulated (Figure 1B). The accumulation of HEMA1 by the FC2 deficiency may increase the global flow of heme biosynthetic pathway and result in the increased heme supply to ER through the FC1 activity in $f_{c} 2-1$. Alternatively, in $f_{c} 2-2$, a decrease in heme supply inside chloroplasts may also lead to the activation of the HEMA1 enzyme because heme can function as a feedback regulator of HEMA1.

\section{FC1 is Involved in Flg22-Dependent Stress Responses}

It is proposed that FC1 is involved in defense response against abiotic and biotic stresses (Singh et al., 2002; Nagai et al., 2007). On the contrary, based on in silico co-expression analysis and flg22 (a 22 amino acid peptide of flagellin, which is a bacterial elicitor) dependent oxidative burst assay, Scharfenberg et al. (2015) proposed that FC2 supplies heme not only for photosynthetic cytochromes, but also for proteins involved in stress responses to bacteria. To verify these hypotheses, we determined the effects of flg22 on the expression of FC1 and FC2. As shown in Figure 8A, FC1 was induced by $1 \mu \mathrm{g} / \mathrm{ml}$ flg22 treatment for $6 \mathrm{~h}$ in wild type and $f c 2-2$, but such induction was not observed in $f c 1-1$. Actually, histochemical analysis of
FC1 showed whole plant induction of FC1 by flg22 treatment (Figure 8A inset). The level of induction was approximately fourfold to untreated control, which is less pronounced than other flg22-responsive genes, such as CYP78, CYP81, and MYB41 (Figure 8B). It should be noted that consistent with previous observation (Nagai et al., 2007), HEMA2 was also induced by the flg22 treatment (Figure 8B).

To further investigate the function of flg22-induced heme production, we determined the peroxidase activities using guaiacol as a substrate. As shown in Figure 8C, peroxidase activities were induced by $0.5 \mu \mathrm{g} / \mathrm{ml} \mathrm{flg} 22$ treatment for $24 \mathrm{~h}$ in wild-type and $f_{c} 2-2$, while such induction was abolished in $f c 1-1$, showing that FC1-producing heme is actually involved in the induction of stress-responsive peroxidase activities. However, when we measured the flg22-induced lignin accumulation, the level of lignin accumulation in $f c 1-1$ was similar to those observed in wild-type and $f_{c} 2-2$ (Figure 8D). Therefore, although the FC1 induction is abolished, the background heme level in $f c 1-1$ may be enough for supplying heme to defense-responsive hemoproteins to synthesize stress-responsive lignin under bacterial infections.

\section{DISCUSSION}

In this study, we analyzed the function of ferrochelatase isoforms using T-DNA insertion mutants of $A$. thaliana. Our phenotype analysis is basically consistent with previous studies (Nagai et al., 2007; Scharfenberg et al., 2015; Woodson et al., 2015), although some discrepancies have been found.

\section{Physiological Importance of FC1-Producing Heme during Development}

In the previous studies, except for the stress-responsive induction, the ubiquitous and light-independent expression of 
A

C
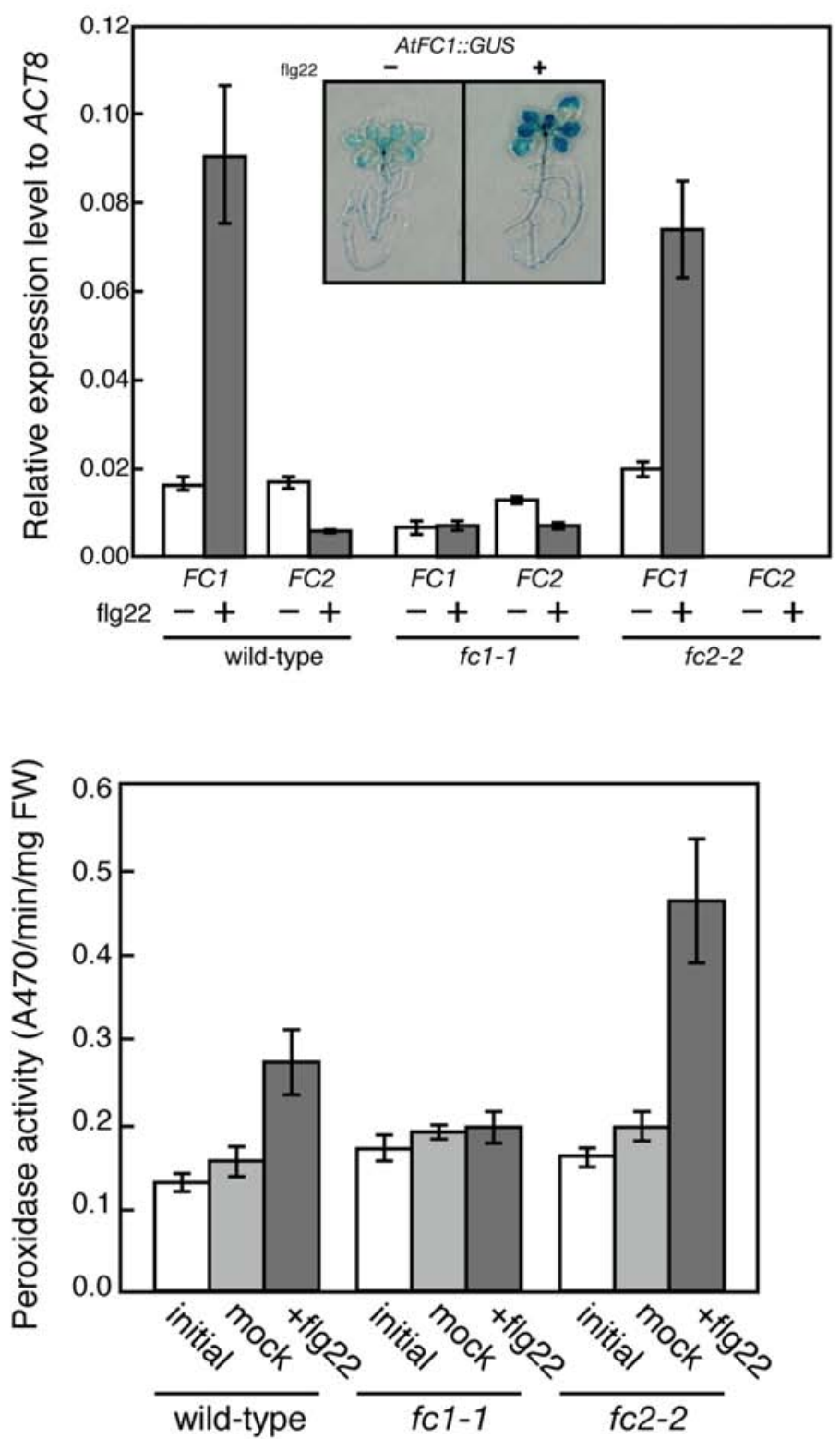

B

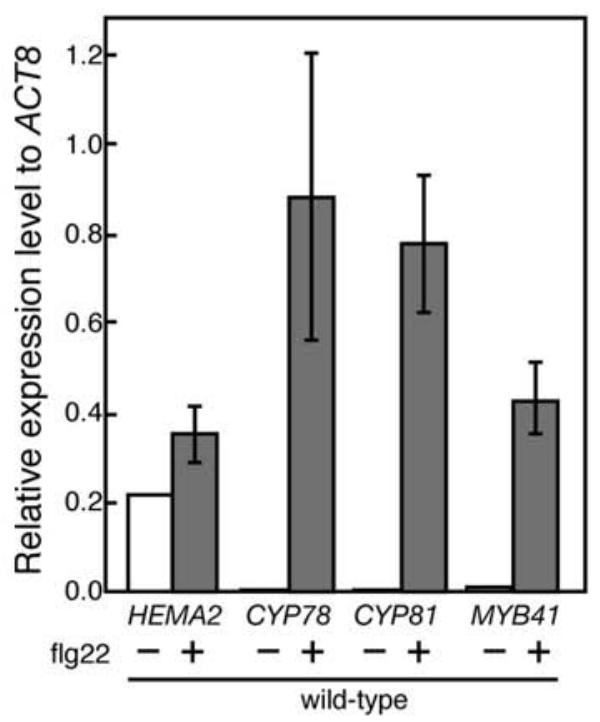

D

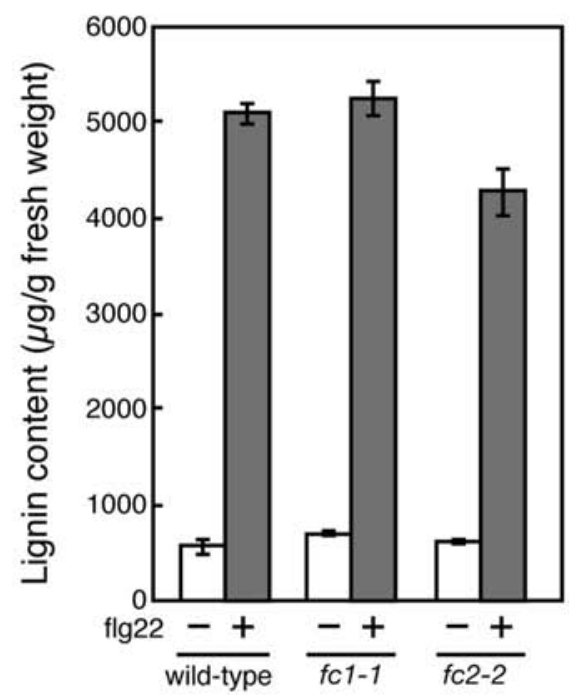

FIGURE 8 | flg22-dependent induction of FC1. (A) qRT-PCR analysis of FC1 and FC2 in wild-type (WT), $f_{C} 1-1$, and $f_{C} 2-2$ treated with $1 \mu \mathrm{g}$ ml ${ }^{-1}$ flg22 for $6 \mathrm{~h}$ after 5 days of culture. Inset is photograph of GUS staining of FC1::GUS line treated with or without flg22. (B) qRT-PCR analysis of stress-responsive genes (HEMA2, CYP78, CYP81, and MYB41) in wild-type treated with $1 \mu \mathrm{g} \mathrm{ml}^{-1}$ flg22 for $6 \mathrm{~h}$ after 5 days of culture. Values are presented as the fold difference from the flg22-untreated wild-type ACTIN8 gene. Bars indicate SEM from three independent experiments. (C) flg22-dependent changes of peroxidase activities. Rosette leaves of 27 -day-old seedlings were vacuum infiltrated with or without $0.5 \mu \mathrm{g} \mathrm{ml}^{-1} \mathrm{flg} 22$ in the presence of $0.001 \%$ Triton X-100. After $24 \mathrm{~h}$ incubation, peroxidase activities were measured by using guaiacol as substrate. (D) Quantification of stress-inducible lignin of wild-type (WT), fc1-1, and fc2-2 seedlings treated with or without $1 \mu \mathrm{g} \mathrm{ml}^{-1}$ flg22 for 3 days after 3 days of culture.

FC1 has been observed under normal conditions tested (Chow et al., 1998; Nagai et al., 2007). Although higher FC1 expression was detected in roots, effect of its deficiency on root growth has not been observed (Figure 2A). Since embryonic lethal phenotype of homozygous $f c 1-2$ was suggested (Woodson et al., 2011), involvement of FC1-produced heme in embryogenesis has been considered. For $f(1-2$, however, we found some of the homozygous seeds can occasionally germinate (Figure 1C). Considering the germinated $f c 1-2$ stopped their growth at initial seedling stage, it suggests that healthy homozygous fc1-2 can occasionally form embryo but seedlings are arrested during further development. Our histochemical analysis of FC1 expression showed prominent staining in primordial tissues of leaves, stipules, and bolting stem in mature seedlings (Figure 3). 
As additional $f c 1-1$ mutation in $f c 2-2$ (homozygous $f c 1-1 \quad f c 2-2$ double mutant) caused developmental arrest before or soon after bolting, FC1-produced heme may also be necessary for newly emerging tissues, such as new leaves and bolting stems.

\section{Involvement of FC2-Producing Heme in Chloroplast Development}

On the contrary, FC2-producing heme is mainly supplied for chloroplast development. Consistent with previous studies, $f c 2$ mutants are abnormally small having pale green rosette leaves with low levels of chlorophylls, carotenoids and several photosynthetic proteins, and their photosynthetic performance was impaired (Scharfenberg et al., 2015; Woodson et al., 2015). Since FC2 deficiency in $f c 2-2$ caused substantial decrease in total heme contents (Figure 2D), it is likely that heme in photosynthetic tissues is predominantly supplied by FC2.

In photosynthetic machineries, FC2-produced heme is mainly incorporated into cytochrome $b_{559}$ and $b_{6} f$ complex. In fact, Scharfenberg et al. (2015) reported that cytochrome $b_{6}$ binding heme was almost undetectable in $f c 2-1$, although reduction of cytochrome $f$ protein was less pronounced. In our study, the $f c 2$ 2 mutant, which is completely deficient in the FC2 expression (Figures 1B and 4B), showed a particular decrease in the cytochrome $f$ level (Figure 4B). Lack of cytochrome $b_{6} f$ complex could strongly affect the intersystem electron transport process between PSII and PSI. Indeed, $f c 2-2$ showed lower qP values, which represents enhanced reduction of the plastoquinone pool, than the wild type and $f_{c 1-1}$ (Figure 6C), although $f_{c 2}-2$ had lower PSII photochemical activity to reduce the plastoquinone pool (Figure 6B). The data suggest a strong retardation of electron transport from the plastoquinone pool to downstream components and consistent with the deficiency of the cytochrome $b_{6} f$ complex in the mutant.

In addition, the $f c 2-2$ mutation increased relative amounts of LHC antennas to reaction center complexes (Figure 4B). Considering the severe reduction of $\mathrm{CP} 43$ and $\mathrm{CP} 47$ in $f c 2-2$, it is likely that the FC2-producing heme is necessary for core antenna complexes of PSII rather than peripheral LHCII antenna complexes. The PAM analyses basically confirmed the results of biochemical analysis of photosynthetic proteins. The analysis revealed that PSII photochemical efficiency represented by $F_{\mathrm{v}} / F_{\mathrm{m}}$ and $F_{\mathrm{v}}^{\prime} / F_{\mathrm{m}}^{\prime}$ was decreased in $f c 2-2$ (Figures $6 \mathrm{~A}, \mathbf{B}$ ), in which energetic disconnection between the PSII reaction center and disassembled LHCII complexes may be involved. Consistently, actual photosynthetic efficiency represented by $\Phi_{\text {II }}$ was considerably decreased in $f c 2-2$ particularly at middle to high PAR (Figure 6A). These changes accompanied increases in thermal dissipation of light energy $\left(\Phi_{\mathrm{NPQ}}\right)$ (Figure 6E), which occurs in the LHCII antenna (Murchie and Niyogi, 2011), as well as quantum yield of non-regulated energy dissipation $\left(\Phi_{\mathrm{NO}}\right)$ (Figure 6E). Accumulation of disassembled LHCII increases dissipation of light energy that cannot be used for photochemical reactions, as fluorescence from the antenna system. Moreover, the increase in relative LHCII levels in $f c 2-2$ may enhance lightinduced NPQ activities. Higher NPQ was also observed in the previous study (Scharfenberg et al., 2015). For the reason of higher NPQ, Scharfenberg et al. (2015) suggested the deficiency of PSBO in fc2-1 may enhance cyclic electron transfer around PSI that induce the abnormally rapid and elevated development of NPQ like the psbo mutant (Allahverdiyeva et al., 2005). Although the involvement of the cyclic electron transfer is not clear, our data suggest that relatively larger size of dysfunctional antenna in $f c 2-2$ may cause higher dissipation of absorbed light energy as heat or fluorescence. Furthermore, deficiency of cytochrome $b_{6} f$ complexes and consequent impairment of intersystem electron transport may increase non-photochemical energy dissipation with reduced $\Phi_{\text {II }}$ in $f c 2-2$.

For the misbalance between PSII core and the peripheral antenna, the impact of the deficiency of cytochrome $b_{559}$ in $f_{c 2}$ 2 on photosynthetic activity should be considered (Figure 4). It is reported that cytochrome $b_{559}$ functions in the cyclic electron chain of PSII to protect PSII from photoinhibition (Stewart and Brudvig, 1998). Although the detailed mechanism remains largely unknown about this complex (Plochinger et al., 2016), the deficiency of heme supply in $f c 2-2$ may affect the function of this complex. Since NPQ involves photoinhibitory component, the possibility that higher NPQ in $f c 2-2$ is related to cytochrome $b_{559}$ deficiency cannot be excluded. On the other hand, it is proposed that among the PSII assembly steps, the formation of cytochrome $b_{559}$-D2 subcomplex is the initial step that serves as a platform for subsequent incorporation of PSII subunits (Nickelsen and Rengstl, 2013). It is possible that the deficiency of cytochrome $b_{559}$ in $f c 2-2$ certainly delayed the PSII assembly of core PSII complex and subsequently resulted in unbalanced accumulation of lower PSII reaction center complexes to LHC antenna.

Alternatively, the possibility that the LHC motif in FC2 is involved in the misbalance between PSII core and the peripheral antenna cannot be excluded. By the presence of the C-terminal LHC motif, FC2 is also categorized as one of eight lightharvesting-like (LIL) proteins in Arabidopsis (Jansson, 1999; Engelken et al., 2010). Unlike the LHC, LIL does not appear to be involved in light harvesting, but some of the LIL proteins appear to be at least temporarily associated with the photosynthetic apparatus (Green et al., 1991; Yao et al., 2007).

\section{FC1-Producing Heme Is Involved in Stress Responses}

In this study, our analysis showed induction of $F C 1$ and repression of FC2 expression by flg22 treatment and abolishment of FC1 induction in $f_{c 1-1}$ (Figure 8A). Furthermore, flg22dependent induction of peroxidase activity was abolished in fc1-1 (Figure 8C). These results are consistent with previous studies (Singh et al., 2002; Nagai et al., 2007). It should be noted that the induction of peroxidase activity was more pronounced in $f c 2-2$ than wild-type, although similar induction of FC1 was observed in this mutant (Figure 8A). High accumulation of hemoproteins was also observed in the ERenriched microsomal LM fraction in $f c 2-2$ (Figure 7). It is possible that such a higher heme flux to extraplastidic organelles is caused by higher HEMA1 level in $f c 2-2$ that increases the global flow of heme biosynthetic pathway since the major FC2-dependent heme production in plastid is prevented in fc2-2. Alternatively, a possibility that a decrease in heme supply inside chloroplasts in $f c 2-2$ may activate HEMA1 activity 
thorough reducing feedback inhibition of heme cannot be excluded.

On the contrary, Scharfenberg et al. (2015) proposed that heme produced by FC2 is involved specifically in response to biotic stress. Concerning the contradictory conclusions about what isoform produces the stress defensive heme, distinct experimental conditions may cause such discrepancy. For flg22 dependent oxidative burst detection, Scharfenberg et al. (2015) used 4- or 5-week old leaf discs and measured immediate response against flg22 treatment within $21 \mathrm{~min}$. In our previous study (Nagai et al., 2007), stress-induced increase of FC1 transcripts was observed at least after $15 \mathrm{~min}$ of wounding treatment. In this sense, FC1 must be firstly induced for production of stress defensive heme. Lower heme levels in $f_{c} 2-1$ may cause higher sensitivity to flg22-dependent oxidative burst (Scharfenberg et al., 2015). Alternatively, the reason why $f c 2-1$ showed reduced levels of oxidative burst may be related to using aged leaves in the assay. In our histochemical assay of FC1, the expression of FC1 in leaves was substantially lowered in 3-weekold plant when compared to 2-week-old plant (Figures 3C,D). Therefore, it is possible that the expression of FC1 in 5-weekold $f_{c} 2-1$ leaves is attenuated to lower levels that are not enough for heme production against flg22 dependent oxidative burst. Considering FC2-produced heme is mainly used for chloroplast development while FC1-produced heme is allocated to extraplastidic locations, it is reasonable to assume that the inductive heme production by FC1 under stress conditions is involved in defense mechanism.

\section{CONCLUSION}

In this study, we showed distinct involvements of FC1 and FC2 in heme supply to subcellular compartments in plant cells. Our data are in accordance with the hypothesis that FC2 produces heme on site for the photosynthetic machinery in the chloroplast and that FC1 is the housekeeping enzyme providing heme cofactor to the entire cell (Nagai et al., 2007; Woodson et al., 2011), but also add new evidence on how heme deficiency in $f c 2-2$ affects the PSII assembly and the FC1-producing heme is actually involved in the defense mechanism against biotic stresses. In addition, our analysis revealed redundant roles of these isoforms. As null $f_{c} 2-2$ becomes greener upon development and is fertile, it is apparent that heme produced by minorly expressing FC1 is partially allocated to plastids and fulfills a sufficient job in the recovery of $f_{c} 2-2$. In contrast, as homozygous $f_{c} 1-2$ could not grow, heme produced by FC2 cannot replace the minor contribution of FC1 on heme production. At present, it is difficult to distinguish whether low but significant accumulation of heme in LM fraction observed in $f c 1-1$ is supplied by remaining FC1or FC2-dependent pathway. If it is solely supplied by FC1, it is possible that FC2-produced heme cannot allocate to ER and possibly nucleus for proposed signaling purpose (Woodson et al., 2011).

Besides the housekeeping function, FC1 is assumed to have defense-related function (Singh et al., 2002; Nagai et al., 2007). In addition to the abiotic stresses (Nagai et al., 2007), our study clearly showed that FC1 is also involved in the defense against biotic stresses, such as pathogenesis. As observed in lignin accumulation (Figure 8D), so far we are not aware of any increased sensitivities to wounding, pathogenesis and other stresses in $f c 1-1$. Since the expression of $F C 1$ is induced by virus infection (Singh et al., 2002), testing of other biotic stress conditions may give further information about FC1 function.

For the regulation of heme allocation, heme trafficking system is also important. Considering that animal mitochondrial ferrochelatase forms complexes with $\mathrm{ABC}$ transporters (Taketani et al., 2003; Chen et al., 2010), distinct binding of each isoform to such transporter protein may occur in plant cell. For heme transfer, heme carrier proteins are also important because of hydrophobic nature of heme. It is demonstrated that heme binding protein (HBP5) interacts with heme oxygenase 1 in plastids probably at the downstream of FC2 in heme catabolic pathway (Hye-Jung et al., 2012), suggesting the existence of specific heme trafficking system. Meanwhile, Vanhee et al. (2011) showed that Golgi-localized TSPO (tryptophan-rich sensory protein) is a heme-binding protein and a potential scavenger of porphyrin via an autophagy-dependent degradation. Further analysis of heme transfer network is necessary in the future.

\section{AUTHOR CONTRIBUTIONS}

NE carried out main experiments of this manuscript. KK contributed photosynthetic characterization and YS performed flg22-responsive assays. NM contributed genetic analysis of $f_{c} 2-2$ mutant. KT and RT performed BN PAGE analysis. TM organized and wrote this manuscript.

\section{FUNDING}

This work was supported by Grants-in-Aids for Scientific Research on Priority Areas (Nos. 24570042, 16K07393 and 26711016).

\section{ACKNOWLEDGMENTS}

We thank Drs. Geraldine Bonnard and Daniele Werck for providing CYP98A3 antibodies, ABRC stock center for providing T-DNA insertion lines and Dr. Kaoru Suzuki for helpful discussions.

\section{SUPPLEMENTARY MATERIAL}

The Supplementary Material for this article can be found online at: http://journal.frontiersin.org/article/10.3389/fpls.2016.01326

FIGURE S1 | (A) Genotyping of $f c 1-1$ fc2-2 double mutant. Primers are depicted in Figure 1A. Since double mutant was infertile, this line is maintained as fc1-1 homozygous and $f c 2-2$ heterozygous seeds. (B) Photograph of each line. Comparison of heterozygous and homozygous lines of double mutant (C), and $f_{C} 2-2$ and homozygous line (D). (E) Typical phenotype of $f_{C} 1-1 f_{C} 2-2$ homozygous double mutant, which stopped its growth before or soon after bolting. 


\section{REFERENCES}

Allahverdiyeva, Y., Mamedov, F., Maenpaa, P., Vass, I., and Aro, E. M. (2005). Modulation of photosynthetic electron transport in the absence of terminal electron acceptors: characterization of the rbcL deletion mutant of tobacco. Biochim. Biophys. Acta 1709, 69-83. doi: 10.1016/j.bbabio.2005.06.004

Arnon, D. (1949). Copper enyzmes in isolated chloroplasts. Polyphenol oxidase in Beta vulgaris. Plant Physiol. 24, 1-5. doi: 10.1104/pp.24.1.1

Bak, S., Beisson, F., Bishop, G., Hamberger, B., Hofer, R., Paquette, S., et al. (2011). Cytochromes p450. Arabidopsis Book 9:e0144. doi: 10.1199/tab.0144

Chen, W., Dailey, H. A., and Paw, B. H. (2010). Ferrochelatase forms an oligomeric complex with mitoferrin-1 and Abcb10 for erythroid heme biosynthesis. Blood 116, 628-630. doi: 10.1182/blood-2009-12-259614

Chow, K. S., Singh, D. P., Walker, A. R., and Smith, A. G. (1998). Two different genes encode ferrochelatase in Arabidopsis: mapping, expression and subcellular targeting of the precursor proteins. Plant J. 15, 531-541. doi: 10.1046/j.1365-313X.1998.00235.x

Engelken, J., Brinkmann, H., and Adamska, I. (2010). Taxonomic distribution and origins of the extended LHC (light-harvesting complex) antenna protein superfamily. BMC Evol. Biol. 10:233. doi: 10.1186/1471-2148$10-233$

Espinas, N. A., Kobayashi, K., Takahashi, S., Mochizuki, N., and Masuda, T. (2012). Evaluation of unbound free heme in plant cells by differential acetone extraction. Plant Cell Physiol. 53, 1344-1354. doi: 10.1093/pcp/pcs067

Green, B. R., Pichersky, E., and Kloppstech, K. (1991). Chlorophyll a/bbinding proteins: an extended family. Trends Biochem. Sci. 16, 181-186. doi: 10.1016/0968-0004(91)90072-4

Hye-Jung, L., Masuda, T., and Buckhout, T. J. (2012). Disrupting the bimolecular binding of the heme binding protein 5 (AtHBP5) with heme oxygenase I (HYI) leads to oxidative stress in Arabidopsis. J. Exp. Bot. 63, 5967-5978. doi: 10.1093/jxb/errs321432

Jansson, S. (1999). A guide to the Lhc genes and their relatives in Arabidopsis. Trends Plant Sci. 4, 236-240. doi: 10.1016/S1360-1385(99) 01419-3

Kobayashi, K., Sasaki, D., Noguchi, K., Fujinuma, D., Komatsu, H., Kobayashi, M., et al. (2013). Photosynthesis of root chloroplasts developed in Arabidopsis lines overexpressing GOLDEN2-LIKE transcription factors. Plant Cell Physiol. 54, 1365-1377. doi: $10.1093 / \mathrm{pcp} / \mathrm{pct} 086$

Kramer, D. M., Johnson, G., Kiirats, O., and Edwards, G. E. (2004). New fluorescence parameters for the determination of $q(a)$ redox state and excitation energy fluxes. Photosyn. Res. 79, 209-218. doi: 10.1023/B:PRES.0000015391.99477.0d

Masuda, T., Suzuki, T., Shimada, H., Ohta, H., and Takamiya, K. (2003). Subcellular localization of two types of ferrochelatase in cucumber. Planta 217, 602-609. doi: 10.1007/s00425-003-1019-2

Masuda, T., and Takahashi, S. (2006). Chemiluminescent-based method for heme determination by reconstitution with horseradish peroxidase apo-enzyme. Anal. Biochem. 355, 307-309. doi: 10.1016/j.ab.2006.04.008

Maxwell, K., and Johnson, G. N. (2000). Chlorophyll fluorescence - a practical guide. J. Exp. Bot. 51, 659-668. doi: 10.1093/jexbot/51.345.659

Melis, A., Spangfort, M., and Andersson, B. (1987). Light-absorption and electron-transport balance between photosystem II and photosystem I in spinach chloroplasts. Photochem. Photobiol. 45, 129-136. doi: 10.1111/j.17511097.1987.tb08413.x

Meskauskiene, R., Nater, M., Goslings, D., Kessler, F., op den Camp, R., and Apel, K. (2001). FLU: a negative regulator of chlorophyll biosynthesis in Arabidopsis thaliana. Proc. Natl. Acad. Sci. U.S.A. 98, 12826-12831. doi: 10.1073/pnas. 221252798

Murashige, T., and Skoog, F. (1962). A revised medium for rapid growth and bioassays with tobacco tissue cultures. Physiol. Plant. 15, 473-497. doi: 10.1111/j.1399-3054.1962.tb08052.x

Murchie, E. H., and Niyogi, K. K. (2011). Manipulation of photoprotection to improve plant photosynthesis. Plant Physiol. 155, 86-92. doi: 10.1104/pp.110.168831

Müsel, G., Schindler, T., Bergfeld, R., Ruel, K., Jacquet, G., Lapierre, C., et al. (1997). Structure and distribution of lignin in primary and secondary cell walls of maize coleoptiles analyzed by chemical and immunological probes. Planta 201, 146-159. doi: 10.1007/Bf01007699
Nagai, S., Koide, M., Takahashi, S., Kikuta, A., Aono, M., Sasaki-Sekimoto, Y., et al. (2007). Induction of isoforms of tetrapyrrole biosynthetic enzymes, AtHEMA2 and AtFC1, under stress conditions and their physiological functions in Arabidopsis. Plant Physiol. 144, 1039-1051. doi: 10.1104/pp.107.100065

Nickelsen, J., and Rengstl, B. (2013). Photosystem II assembly: from cyanobacteria to plants. Annu. Rev. Plant Biol. 64, 609-635. doi: 10.1146/annurev-arplant050312-120124

Nozaki, M., Sugiyama, M., Duan, J., Uematsu, H., Genda, T., and Sato, Y. (2012). A missense mutation in the glucosamine-6-phosphate N-acetyltransferaseencoding gene causes temperature-dependent growth defects and ectopic lignin deposition in Arabidopsis. Plant Cell 24, 3366-3379. doi: $10.1105 /$ tpc. 112.102806

Plochinger, M., Schwenkert, S., von Sydow, L., Schroder, W. P., and Meurer, J. (2016). Functional update of the auxiliary proteins PsbW, PsbY, HCF136, PsbN, TerC and ALB3 in maintenance and assembly of PSII. Front. Plant Sci. 7:423. doi: $10.3389 /$ fpls.2016.00423

Sato, Y., Sugiyama, M., Górecki, R. J., Fukuda, H., and Komamine, A. (1993). Interrelationship between lignin deposition and the activities of peroxidase isoenzymes in differentiating tracheary elements of Zinnia: analysis using $\mathrm{L}$ - $\alpha$-aminooxy- $\beta$-phenylpropionic acid and 2-aminoindan-2-phosphonic acid. Planta 189, 584-589. doi: 10.1007/BF00198223

Sato, Y., Yajima, Y., Tokunaga, N., and Whetten, R. (2011). Comparison between tracheary element lignin formation and extracellular lignin-like substance formation during the culture of isolated Zinnia elegans mesophyll cells. Biologia 66, 88-95. doi: 10.2478/s11756-010-0130-7

Scharfenberg, M., Mittermayr, L., Von Roepenack-Lahaye, E., Schlicke, H., Grimm, B., Leister, D., et al. (2015). Functional characterization of the two ferrochelatases in Arabidopsis thaliana. Plant Cell Environ. 38, 280-298. doi: $10.1111 /$ pce. 12248

Schenke, D., Bottcher, C., and Scheel, D. (2011). Crosstalk between abiotic ultraviolet-B stress and biotic (flg22) stress signalling in Arabidopsis prevents flavonol accumulation in favor of pathogen defence compound production. Plant Cell Environ. 34, 1849-1864. doi: 10.1111/j.1365-3040.2011.02381.x

Singh, D. P., Cornah, J. E., Hadingham, S., and Smith, A. G. (2002). Expression analysis of the two ferrochelatase genes in Arabidopsis in different tissues and under stress conditions reveals their different roles in haem biosynthesis. Plant Mol. Biol. 50, 773-788. doi: 10.1023/A:1019959224271

Sobotka, R., Tichy, M., Wilde, A., and Hunter, C. N. (2010). Functional assignments for the C-terminal domains of the ferrochelatase from synechocystis PCC 6803: the $\mathrm{CAB}$ domain plays a regulatory role and region II is essential for catalysis. Plant Physiol. 155, 1735-1747. doi: 10.1104/pp.110.167528

Stewart, D. H., and Brudvig, G. W. (1998). Cytochrome b559 of photosystem II. Biochim. Biophys. Acta 1367, 63-87. doi: 10.1016/S0005-2728(98)00139-X

Suzuki, T., Masuda, T., Singh, D. P., Tan, F. C., Tsuchiya, T., Shimada, H., et al. (2002). Two types of ferrochelatase in photosynthetic and nonphotosynthetic tissues of cucumber: their difference in phylogeny, gene expression, and localization. J. Biol. Chem. 277, 4731-4737. doi: 10.1074/jbc.M105613200

Takahashi, K., Takabayashi, A., Tanaka, A., and Tanaka, R. (2014). Functional analysis of light-harvesting-like protein 3 (LIL3) and its light-harvesting chlorophyll-binding motif in Arabidopsis. J. Biol. Chem. 289, 987-999. doi: 10.1074/jbc.M113.525428

Takahashi, S., and Masuda, T. (2009). High throughput heme assay by detection of chemiluminescence of reconstituted horseradish peroxidase. Comb. Chem. High Throughput Screen. 12, 532-535. doi: 10.2174/138620709788489028

Taketani, S., Kakimoto, K., Ueta, H., Masaki, R., and Furukawa, T. (2003). Involvement of $\mathrm{ABC7}$ in the biosynthesis of heme in erythroid cells: interaction of ABC7 with ferrochelatase. Blood 101, 3274-3280. doi: 10.1182/blood-200204-1212

Tanaka, R., Kobayashi, K., and Masuda, T. (2011). Tetrapyrrole metabolism in Arabidopsis thaliana. Arabidopsis Book 9:e0145. doi: 10.1199/tab.0145

van Kooten, O., and Snel, J. F. (1990). The use of chlorophyll fluorescence nomenclature in plant stress physiology. Photosynth. Res. 25, 147-150. doi: 10.1007/BF00033156

Vanhee, C., Zapotoczny, G., Masquelier, D., Ghislain, M., and Batoko, H. (2011). The Arabidopsis multistress regulator TSPO is a heme binding membrane protein and a potential scavenger of porphyrins via an autophagy-dependent degradation mechanism. Plant Cell 23, 785-805. doi: 10.1105/tpc.110. 081570 
Wittig, I., Braun, H.-P., and Schägger, H. (2006). Blue native PAGE. Nat. Protoc. 1, 418-428. doi: 10.1038/nprot.2006.62

Woodson, J. D., Joens, M. S., Sinson, A. B., Gilkerson, J., Salome, P. A., Weigel, D., et al. (2015). Ubiquitin facilitates a quality-control pathway that removes damaged chloroplasts. Science 350, 450-454. doi: 10.1126/science.aac7444

Woodson, J. D., Perez-Ruiz, J. M., and Chory, J. (2011). Heme synthesis by plastid ferrochelatase I regulates nuclear gene expression in plants. Cur. Biol. 21, 897-903. doi: 10.1016/j.cub.2011.04.004

Yao, D., Kieselbach, T., Komenda, J., Promnares, K., Prieto, M. A. H., Tichy, M., et al. (2007). Localization of the small CAB-like proteins in photosystem II. J. Bio. Chem. 282, 267-276. doi: 10.1074/jbc.M605463200
Conflict of Interest Statement: The authors declare that the research was conducted in the absence of any commercial or financial relationships that could be construed as a potential conflict of interest.

Copyright (C) 2016 Espinas, Kobayashi, Sato, Mochizuki, Takahashi, Tanaka and Masuda. This is an open-access article distributed under the terms of the Creative Commons Attribution License (CC BY). The use, distribution or reproduction in other forums is permitted, provided the original author(s) or licensor are credited and that the original publication in this journal is cited, in accordance with accepted academic practice. No use, distribution or reproduction is permitted which does not comply with these terms. 